\title{
Motivación por el aprendizaje de las ciencias naturales, en los estudiantes de básica primaria del centro educativo, cuatro bocas, municipio de San Martín, Cesar
}

\section{Motivation for learning of natural sciences in elementary school students from cuatro bocas educational center, municipality of san martin, cesar}

\section{Motivação para aprendizagem da natural ciência na atenção primária estudantes basic educacional centro, município de quatro gargalo de san martin, cesar}

\author{
Magaly Gómez-Castilloํㅡ, Carlos Sebastián Gómez-Vergel ${ }^{2}$, Mawency Vergel-Ortega ${ }^{3}$
}

Forma de citar: Gómez-Castillo, M., Gómez-Vergel, C. \& Vergel-Ortega, Motivación por el aprendizaje de las ciencias naturales, en los estudiantes de básica primaria del centro educativo, cuatro bocas, municipio de San Martín, Cesar, Revista EcoMat. 7[101-111].

Recibido:

Agosto 30 de 2015

Aceptado:

Diciembre 6 de 2015

\section{Resumen}

El artículo busca evaluar el grado de motivación y creatividad de los estudiantes de básica primaria del Centro educativo cuatro bocas frente al aprendizaje de las ciencias naturales. La investigación siguió un enfoque cuantitativo, descriptivo, utiliza escalas de motivación teniendo en cuenta indicadores del Ministerio de Educación Nacional. Resultado: Un 17, $9 \%$ califica como alta la motivación por aprender ciencias naturales, entre 1 y 7 se ubica la escala de motivación, donde la variable prestigio tiene los valores más bajos, éxito y objetivo así como el norte para elevar la motivación y creatividad tienen los valores más altos. 4 es el valor medio de motivación que los estudiantes dan a las diferentes variables asociadas a la motivación. Conclusión: variables invención, utilidad, objetivos y disfrutar el aprendizaje se asocia a mejora de la motivación de los estudiantes.

Palabras clave: ciencias, creatividad, experimentación, motivación.

${ }^{1}$ Licenciada en Educación Básica, ciencias naturales y educación ambiental

Mag1103@hotmail.com Centro Educativo Cuatro Bocas,

San Martín, Colombia

${ }^{2}$ Candidato Bachiller carlossetox@gmail.com Colegio Calasanz Cúcuta- Colombia

${ }^{3}$ Doctora en Educación mawency@ufps.edu.co Universidad Francisco de Paula Santander, Cúcuta-Colombia

\begin{abstract}
The article evaluates the degree of motivation and creativity of the elementary school students of Four Mouths Educational Center facing the learning of natural sciences. The research followed a quantitative, descriptive approach, using motivational scales taking into account indicators from the Ministry of National Education. Results: 17, 9\% rate the motivation to learn natural sciences as high, scale is motivation located between 1 and 7, where the prestige variable has the lowest values, while success and objective as well as the north to raise motivation and creativity have the highest values. 4 is the average value of motivation that students give to the different variables associated with motivation. Conclusion: variables invention, utility, objectives, and learning joy is associated with improved motivation of students.
\end{abstract}

Keywords: Motivation, experimentation, science, creativity. 
No. 1

Enero-Diciembre 2016 ISSN 1794-8231 E-ISSN $2462-8794$ PP: $101-111$

\begin{abstract}
Resumo
O artigo procura avaliar o grau de motivação e criatividade dos alunos do Centro Educacional quatro pescoço primário em relação à aprendizagem das ciências naturais. A pesquisa seguiu um quantitativas escalas, descritivos usados abordagem motivacional tendo em conta os indicadores do Ministério da Educação. Resultado: A 17, 9\% qualifica como alta motivação para aprender ciência, entre 1 e 7 da escala de motivação, onde a variável prestígio tem o menor valores, o sucesso e objectiva e norte para aumentar a motivação reside e criatividade têm os maiores valores. 4 é o valor médio de motivação variáveis students'value diferentes motivação associados. Conclusão: variáveis invenção, utilidade, e desfrutar de objectivos de aprendizagem está associada com aumento da motivação dos alunos.
\end{abstract}

Palavras-chave: motivação, experimentação, ciência, criatividade.

\section{Introducción}

La enseñanza de las ciencias naturales en la educación, siempre ha sido motivo de análisis desde el punto de vista pedagógico. El ejercicio de la profesión de un maestro en ciencias naturales, debe tener presente que su misión ante todo, no puede ser otra, que la de formar y orientar al niño, niña, joven en su aprendizaje, en la búsqueda de la verdad y la generación de conocimiento (Vergel, Martínez \& Zafra, 2015). Además de impartir sus conocimientos en esa área, con la máxima eficiencia y probidad, un maestro debe motivar, enseñarles a crear y a disfrutar la ciencia, entendiendo que los mismos, deben constituir, analizar y decidir cómo transformar su realidad y su región a partir del conocimiento que adquieren y generan, a fin de que a través de las enseñanzas impartidas, se logre promover con altos estándares de calidad, el desarrollo del pensamiento crítico y creativo, no sólo en el aula de clase, sino para la vida (Ortiz,2012)

A partir de las observaciones se generan preguntas y racionamientos se construyen hipótesis se deducen principios y se elaboran leyes generales y sistemas organizados por medio de un método científico. Método científico que está conformado por un conjunto de normas y pasos que le otorgaran validez y rigor científico al proceso de investigación en este sentido sus hallazgos y observaciones deberá ser siempre objetivos y comprobables las hipótesis planteadas se ponen a prueba mediante experimentación para generar resultados que demuestren $o$ refutan la hipótesis formulas o conclusiones que puede llevar a replantear nuevas hipótesis (Martínez, Vergel \& Zafra, 2015).

De otra parte, la motivación por otra parte, juega un papel fundamental en el aprendizaje, es conocida la constante preocupación de los profesionales de la enseñanza por la falta de motivación en los alumnos no sólo para que estudien sino para que aprendan, hagan suyo el conocimiento y de esa forma desarrollen su personalidad (Alonso, 1984; Dinello(2006); Sanmartín, Carbonell, \& Baños(2011); Martin(2011); Pelechano(1972), Mitjans \& Díaz, 2013; Ruiz \& Massa(2011) Sánchez(2010). (Martínez, Vergel \& Zafra, 2015).

En el ámbito internacional, la enseñanza de las Ciencias Naturales estuvo marcada por limitaciones en la recepción de un cúmulo de definiciones que evitaban dar lugar al pensamiento crítico. Por mucho tiempo se ignoró que los alumnos tenían experiencias 
propias y por lo tanto traían consigo sus propias definiciones, dado que el modelo que se imponía en las aulas, se apoyaba en el método expositivo y la clase magistral, relegando de esta forma a los alumnos, a una situación de espectador pasivo (Vergel \& Largo ,2015), desmotivado con ansias de realizar otras actividades diferentes a asistir a la escuela, actividades que le produjeran gozo o dinero (Zafra, Martínez \& Vergel ,2014).

En el ámbito nacional, investigadores señalan que las dificultades para el aprendizaje de las ciencias naturales, están altamente relacionadas con los siguientes aspectos comprensión del lenguaje tecnológico, comprensión de los símbolos y esquemas, comprensión de los conceptos abstractos de la asignatura, la relación entre la realidad científica (el aprendizaje en el aula) y la cotidianidad. Posiblemente esta problemática que presentan los estudiantes, se deriva de la poca o nula relación que se establece entre el nuevo conocimiento, sus concepciones previas, la vida cotidiana y las experiencias laborales (Vergel, Gallardo \& Martínez, 2014).

Así mismo, estudiantes de instituciones de educación básica y media manifiestan que los diversos fenómenos físicos y químicos son poco perceptibles por nuestros sentidos y el esfuerzo intelectual para comprender la asignatura, se hace más complejo debido a que los textos de enseñanza, utilizan símbolos y esquemas para representar las ideas que poco aportan a la comprensión de la asignatura misma (Vergel \& Gallardo, 2007). Se ha observado que en el Centro Educativo Cuatro Bocas, los estudiantes tienen una actitud de apatía y desinterés frente a algunas asignaturas, apatía que se manifiesta mediante conductas que los profesores de las diversas áreas, perciben a través de diferentes actitudes Rincón(2011), parece ser que estas reacciones obedecen a un problema grave de metodología, dado el hecho de que generalmente muchos de los alumnos afectados por esta causa, se refugien en el anonimato del aula de clase, al sentirse incapaces de comprender lo que el profesor intenta enseñarle.

Teniendo en cuenta lo anterior, en la investigación, se buscó analizar la influencia deldesarrollo de la creatividad en la motivación de los niños y niñas por el aprendizaje de las ciencias. Los contenidos tendrán vinculación con las ansias de motivación de los estudiantes, relacionadas con el conocimiento y la exploración del mundo, la experimentación, trabajo en el campo a partir de sembrado, arado u observación de especies (Vergel, 2015) además de una progresiva apropiación de algunos modelos y/o teorías propios de las ciencias naturales, para interpretar y explicar la naturaleza.

\section{Diseño Metodológico}

Desde una visión epistemológica el proyecto seguirá el enfoque cuantitativo, orientado a la explicación descriptiva de casos, la comprensión y a la transformación de la realidad objeto de estudio; apoyado en el enfoque cualitativo siguiendo planteamientos de Campos (2009), “combinar varios enfoques de los que aprovecha sus fortalezas y minimiza sus debilidades. Se puede decir que se ubica en el punto medio de una línea continua, donde los otros dos métodos se ubican en los extremos", el diseño metodológico se utilizaron los parámetros de sigue un diseño de tipo campo. Se trabajó con una población treinta (38) estudiantes de la sede Cuatro Bocas del grado sexto jornadas a noveno jornada de la mañana. La muestra intencional, la constituyeron 30 estudiantes que respondieron todo los test e instructivos y finalizaron el año escolar. Según Balestrini (1998), se concibe como población "cualquier conjunto finito o infinito de personas, casos o elementos que presentan características comunes" (p.123).
Enero-Diciembre 2016 ISSN 1794-8231 E-ISSN 2462 - 8794 PP: 101-111 
No. 1
Las variables a considerar están relacionadas con el dispositivo de medición aplicado a docentes, estudiantes, los programas académicos, su pertinencia, productividad, en escalas que van desde la nominal hasta la de razón, pasando por la ordinal y la de intervalo. (Vergel, Martínez, \& Duarte, 2015). La operacionalización de variables implica la descripción de indicadores generales teniendo en cuenta indicadores de gestión del Departamento Administrativo de la función Pública, indicadores SUE, indicadores del ministerio de Educación Nacional para universidades e indicadores del Observatorio Laboral, indicadores de motivación son creatividad, grado de creatividad, base motivacional, altruismo, colectivismo, tipo de (motivación integradora, instrumental, intrínseca, extrínseca, dominante) Las Dimensiones a considerar son la interacción social y las relaciones interpersonales. (Huertas, Esmeral \& Sánchez, 2014) La variable Independiente será la empatía y variables dependiente: Rendimiento académico de los estudiantes, competencias en área de ciencias.

El cuestionario se definió como un conjunto de preguntas sobre un tema o un grupo de temas elaborados para que una persona las conteste (Richards, Platt \& Platt,H. 1997). Además, estos autores señalan que los cuestionarios se emplean en muchas ramas de la lingüística aplicada, como en los estudios de uso de la lengua, el estudio de actitudes y motivación y en el análisis de necesidades.

Se toma como referente inicial Test de Maale, en cada una de las afirmaciones se dan 5 opciones graduales en torno al parámetro valorativo descrito a continuación: motivación dominante con un máximo de puntaje es 20, para averiguar el grado de motivación de los se suman los factores deseo, interés, esfuerzo (Gardner, 1985). Se suma el resultado de las 12 preguntas que tienen un mínimo de 1 punto y un máximo de 5 puntos $(5 \times 12=$
60). Un Cuarto apartado está compuesto por los componentes tanto humanos: profesor y grupo de compañeros, como no humanos curso y entorno didáctico de la situación del aprendizaje; seis ítems revelan la actitud que los aprendientes tienen hacia su profesorado. Se toman en cuenta tanto aspectos humanos como pedagógicos.

Se utilizan además escalas de diferencial semántico para conocer la actitud de los informantes hacia el grupo de compañeros y hacia el curso respectivamente. Se ordenan aleatoriamente los pares de adjetivos polares evitando que los positivos o los negativos aparezcan siempre en la misma columna, tal como sugiere Vergel, Orjuela \& Martínez, (2014), para impedir que los informantes marquen mecánicamente el mismo espacio. Dado que las escalas utilizadas dan 7 posibilidades de elección para expresar la opinión, los valores numéricos son de un mínimo de 1 para los adjetivos negativos y de un máximo de 7 para los positivos respectivamente por lo que el valor máximo que se puede alcanzar en esta parte es de 35 $(5 \times 7=35)$. Se aplica una escala de intensidad en forma de cuadrícula para averiguar la actitud de los aprendientes hacia el entorno didáctico o contexto en general (libro, material didáctico, número de participantes, mobiliario, aula, entre otros). Los informantes pueden valorar cada uno de los aspectos del entorno con elementos graduales de muy bueno a muy malo. El grado de fiabilidad se reflejó en el coeficiente alfa de Cronbach $=0.8$

La fase diagnóstico comprende entonces la caracterización de niños y niñas a través de encuesta. Implementación de test de creatividad, e implementación de test de motivación. Se realiza una medición en el aula a los estudiantes, incluyendo desde el grado $6^{\circ}$ hasta $9^{\circ}$ guardando la confidencialidad del mismo mediante la aplicación de un operador neutral y sin identificación de las entrevistas. Los datos primarios de las 
puntuaciones fueron sometidos a la prueba de normalidad en los dos factores estudiados (año y género), se realizará prueba de análisis de varianzas, estimación de estadígrafos descriptivos, en todos los factores y sus niveles correspondientes. (Cascón, 2000)

\subsection{Población objeto de estudio}

La población objeto de estudio estuvo comprendida por 30 estudiantes con edades entre 11 y 17 años, promedio de edad 12.97 años, $50 \%$ lo constituyen jóvenes de género masculino y el $50 \%$ de género femenino. $36.8 \%$ (14) de grado sexto, $23.7 \%$ (9) de grado séptimo, $23.7 \%$ de grado octavo, $15.8 \%$ (6) de grado noveno. El valor medio en creatividad inicial (primer período) es de 6 puntos, $15 \%$

Tabla I. La motivación por aprender ciencias naturales

\begin{tabular}{|l|l|l|l|r|c|}
\hline \multicolumn{2}{|c|}{} & Frecuencia & Porcentaje & Porcentaje válido & $\begin{array}{c}\text { Porcentaje } \\
\text { acumulado }\end{array}$ \\
\hline \multirow{3}{*}{ Válidos } & Medio & 11 & 39,3 & 40,7 & 40,7 \\
\cline { 2 - 6 } & Alto & 8 & 28,6 & 29,6 & 70,4 \\
\cline { 2 - 6 } & Muy alto & 5 & 17,9 & 18,5 & 88,9 \\
\cline { 2 - 6 } & Aprender & 3 & 10,7 & 11,1 & 100,0 \\
\cline { 2 - 6 } & Total & 27 & 96,4 & 100,0 & \\
\hline Perdidos & Sistema & 1 & 3,6 & & \\
\hline Total & 28 & 100,0 & & \\
\hline
\end{tabular}

En general la motivación que sienten por el estudio los jóvenes es alto (46.4\%). Un 17, $9 \%$ califica como alta la motivación por aprender ciencias naturales, como regular un $32,1 \%$, y 21,45 como buena. Un $17,9 \%$ tiene alto concepto de sí mismo como estudiante, $32,1 \%$ considera que es regular. El amor por la escuela y compañeros es alto y muy alto para un 50\%. Asociado a aprender. Un 71,45 considera que sus calificaciones están en un nivel medio, un $25 \%$ en un nivel alto. Se observa un alto grado de discriminación en la escuela. Un 39.3\% considera que el juego aporta conocimiento, sirve de estrategia para el desarrollo del conocimiento. Un $82,1 \%$ califica como alto y muy alto el desempeño de la profesora de ciencias, mientras un 3,6\% (1 estudiante) lo califica como bajo. Un $82,1 \%$ califica como alto y muy alto el desempeño de la profesora de ciencias, mientras un valora con puntajes de 2 y $75 \%$ con valores de 10 puntos. Sin embargo un porcentaje de estudiantes valora con puntajes superiores a 10 en la escala Análisis de resultados en test de creatividad en el segundo período, muestra que el valor que más obtuvo porcentaje dentro de los encuestados es de 15.8, el 50\% de los estudiantes obtuvieron un puntaje de 16.5. El $25 \%$ de los estudiantes obtuvieron puntajes menores o iguales a 14.25 , que correspondería a personas algo creativas. El $75 \%$ de los estudiantes obtuvieron puntajes menores o iguales a 17.7, que correspondería a personas creativas. De otra parte, Como buena, responsable, inteligente, amable es descrita la profesora de ciencias. (Tabla I).
3,6 . Inglés es la asignatura que menos les gusta. Asignatura de mejor desempeño es matemáticas para un $25 \%$, artística para un $21,4 \%$, sociales $(10,7 \%)$, biología $(10,7 \%)$. Inglés es la asignatura de más bajo desempeño manifiesto por un $57,1 \%$. Un $7,1 \%$ piensa algunas veces en dejar la escuela. Un $82,1 \%$ no lo ha considerado. Un $64,3 \%$ tiene una imagen de éxito asociado a ser un gran profesional, el $17,9 \%$ lo asocia a ser excelente, y un $14,3 \%$ a un buen futuro. El $39,3 \%$ considera que su nivel de inteligencia es básico, un $28,6 \%$ alto.

\begin{tabular}{|c|c|}
\hline Porcentaje validido & $\begin{array}{c}\text { Porcentaje } \\
\text { acumulado }\end{array}$ \\
\hline 40,7 & 40,7 \\
\hline 22,6 & 70,4 \\
\hline 18,5 & 88,9 \\
\hline 11,1 & 100,0 \\
\hline 100,0 & \\
\hline & \\
\hline
\end{tabular} ISSN 1794-8231 -ISSN $2462-8794$ PP: $101-111$

Referente a cómo desean las clases de ciencia, un $21,4 \%$ considera que deben ser creativas, $14,3 \%$ con laboratorios, un $10,7 \%$ verla dos veces a la semana, $7,1 \%$ más científicas, mejorando la relación con la profesora, mas explicada. (Acevedo, Vergel \& Florez, 2012) 
No. 1

Enero-Diciembre 2016 ISSN 1794-8231 E-ISSN $2462-8794$ PP: $101-111$
Motivación por el aprendizaje de las ciencias naturales, en los estudiantes de básica primaria del centro educativo, cuatro bocas, municipio de San Martín, Cesar

Frente a profesión que les gustaría desempeñar, mencionan un 17,9\% médico, 10,7\% veterinario, $10,7 \%$ ingeniero civil, 7,15 ambiental, artística (7,1\%), otras profesiones mencionadas son futbolista, profesor, mecánico, paleontólogo, ingeniero de sistemas, diseñador, biólogo marino, francotirador. Experimentos, videos, aplicación a la vida son aspectos que los motivan en clase de ciencias. Profesora y asignatura de mejor desempeño son variables a tener en cuenta en metodología a implementar para mejorar la motivación. (Tabla II).

\section{Resultados}

\subsection{Test Motivación}

Tabla II. Promedio

\begin{tabular}{|c|c|c|c|c|c|}
\hline \multicolumn{2}{|c|}{} & Frecuencia & Porcentaje & Porcentaje válido & Porcentaje acumulado \\
\hline \multirow{6}{*}{} & 65 & 3 & 7,9 & 7,9 & 7,9 \\
\cline { 2 - 6 } & 69 & 1 & 2,6 & 2,6 & 10,5 \\
\cline { 2 - 6 } & 70 & 6 & 15,8 & 15,8 & 26,3 \\
\cline { 2 - 6 } & 71 & 2 & 5,3 & 5,3 & 31,6 \\
\cline { 2 - 6 } & 72 & 4 & 10,5 & 10,5 & 42,1 \\
\cline { 2 - 6 } & 73 & 4 & 10,5 & 10,5 & 52,6 \\
\cline { 2 - 6 } & 74 & 6 & 15,8 & 15,8 & 68,4 \\
\cline { 2 - 6 } & 75 & 2 & 5,3 & 5,3 & 73,7 \\
\cline { 2 - 6 } & 76 & 1 & 2,6 & 2,6 & 76,3 \\
\cline { 2 - 6 } & 77 & 1 & 2,6 & 2,6 & 78,9 \\
\cline { 2 - 6 } & 78 & 2 & 5,3 & 5,3 & 94,2 \\
\cline { 2 - 6 } & 80 & 4 & 10,5 & 10,5 & 100,0 \\
\cline { 2 - 6 } & 85 & 2 & 5,3 & 5,3 & \\
\cline { 2 - 6 } & Tot & 38 & 100,0 & 100,0 & \\
\hline
\end{tabular}

Fuente: autores

Los puntajes de escala total de motivación inicial se distribuyeron en valores entre 65 y 85 . (Ver Figura 1).

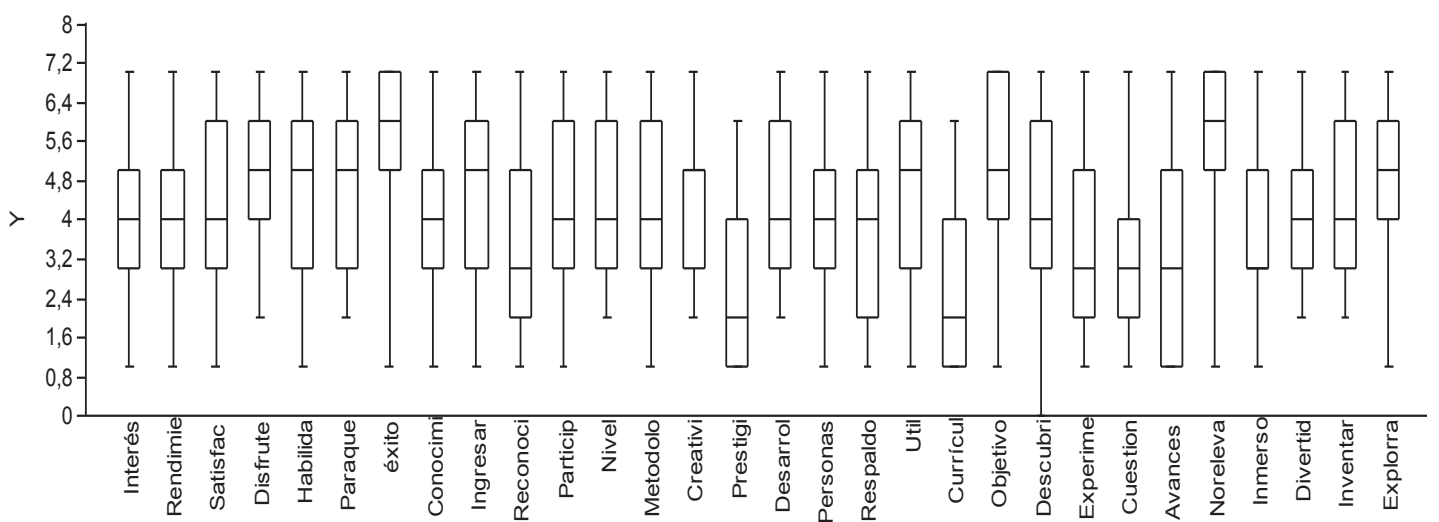

Figura 1. Diagrama de caja, variables asociadas a motivación Fuente: autores

Entre 1 y 7 se ubica la escala de motivación, donde la variable prestigio tiene los valores más bajos, éxito y objetivo así como el norte para elevar la motivación y creatividad tienen los valores más altos. 4 es el valor medio de motivación que los estudiantes dan a las diferentes variables asociadas a la motivación. (Ver Figura 2). 


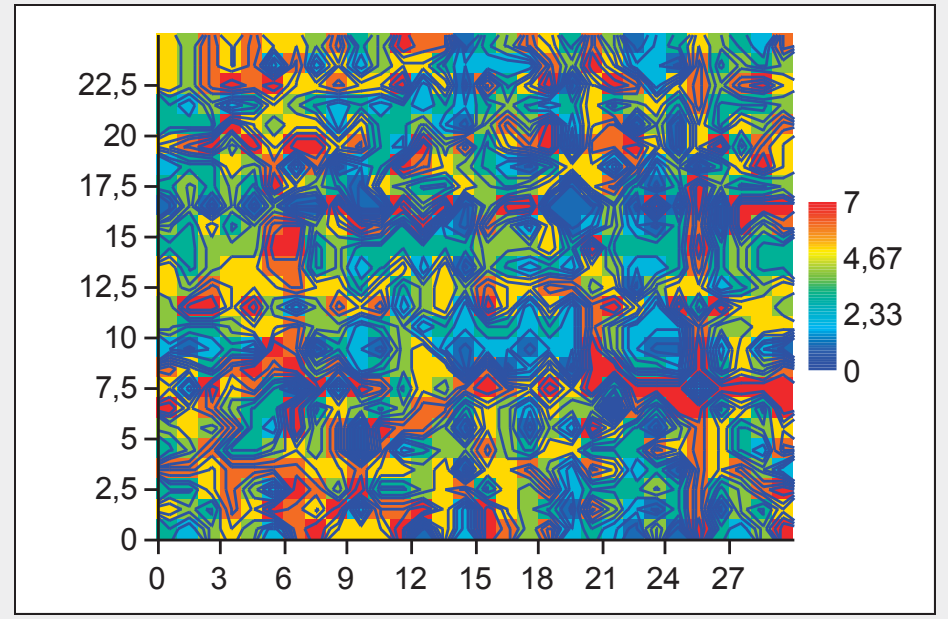

Enero-Diciembre 2016 ISSN 1794-8231

Figura 2. Variabilidad diagrama de calor de motivación Fuente: autores

Existe alta variabilidad en torno a la motivación de los estudiantes por el aprendizaje de las ciencias. La normalización muestra datos asociados a dimensión edad y promedio dentro de lo normal, solo se observa un dato atípico. (Ver Figura 3).

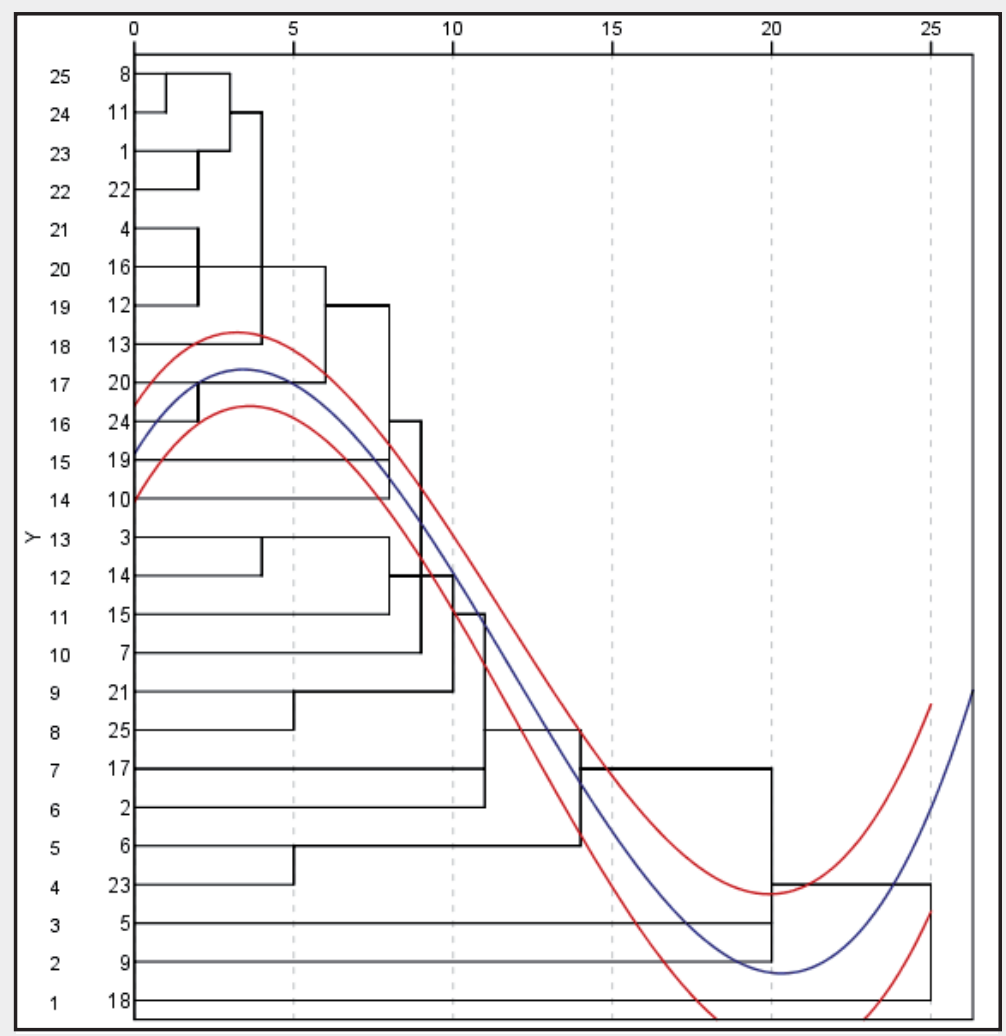

Figura 3. Dendograma y asociación de estudiantes antes y después de mejora en motivación Fuente: autores

El grado de los jóvenes y su distribución y caracterísiticas sociales explican en un 39.8\% puntajes en motivación. Se observa la conformación de tres conglomerados distribuyendo lo jóvenes altamente asociados según puntaje en escala motivación como estudiantes $8,11,122$, $4,16,12,13,20,24,19$ y 10 ; asi mismo un segundo conglomerado con asociatividad alta son 3 , 
No. 1
Motivación por el aprendizaje de las ciencias naturales, en los estudiantes de básica primaria del centro educativo, cuatro bocas, municipio de San Martín, Cesar

14, 15,7,21,25,17 y 2, 6 y 23. Y estudiantes no asociados a los demás son 5,9,18. Los jóvenes se distribuyen según interés y habilidad en ciencias, organizados en cuatro conglomerados, correspondiendo con analisis factorial y componentes principales. (Ver Figura 4).

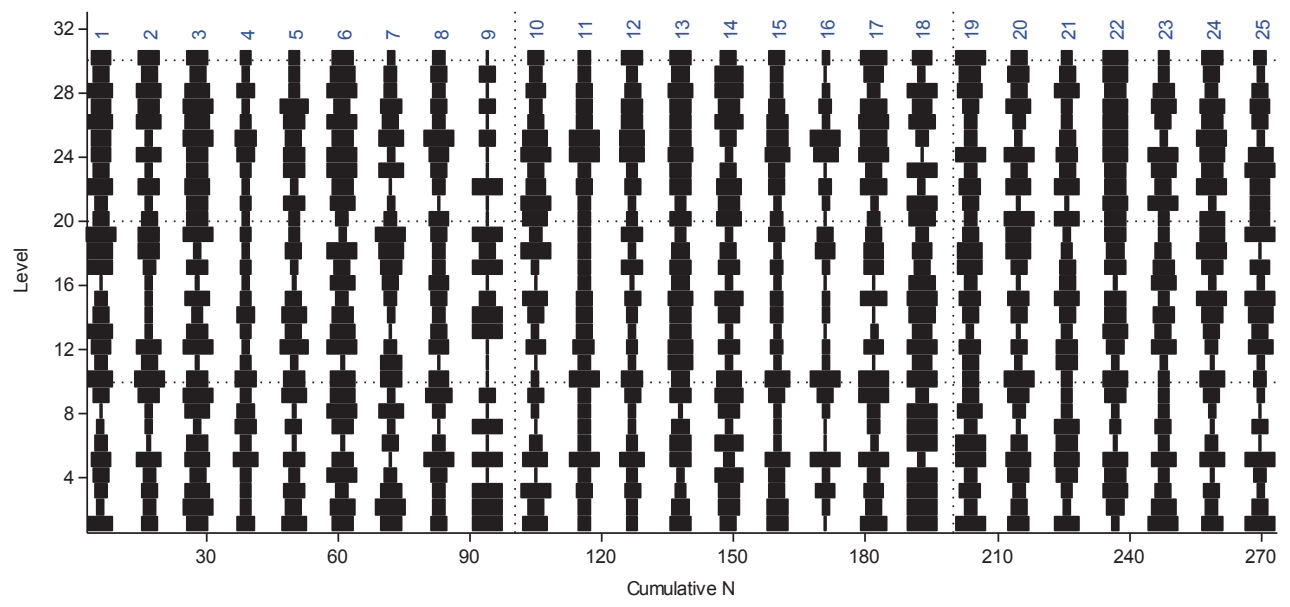

Figura 4. Valor y nivel de motivación acumulado por ítem

Fuente: autores

Solo se observa un caso de un estudiante que no tuvo variabilidad en motivación, caso estudiante 16. Los estudiantes inicialmente se cuestionan sobre para qué sirve aprender ciencias, dando una valoración baja a estos ítems, así mismo puntajes bajos se obtienen en no leo porque mis compañeros consideran que no es relevante, por prestigio de científicos. Dan alto valor antes y después de implementadas metodologías para mejorar motivación a herramientas para explorar, inventar, aporte y metodología de la maestra. (Ver Tabla III).

Tabla III. Porcentaje de estudiantes que califican

\begin{tabular}{|l|l|l|}
\hline & Período I & Período III \\
\hline $\begin{array}{l}\text { Agrado por aprender ciencias } \\
\text { naturales }\end{array}$ & $17,9 \%$ alto & $82.1 \%$ alto \\
\hline Metas & $57,1 \%$ & $71,5 \%$ \\
\hline $\begin{array}{l}\text { Calificaciones y desempeño en } \\
\text { ciencias }\end{array}$ & $32.1 \%$ alto & $64.3 \%$ alto \\
\hline Desempeño profesora & $82,1 \%$ alto & $98 \%$ alto \\
\hline
\end{tabular}

Fuente: autores

Se observa que variables que caracterizan el aprendizaje de las ciencias en los jóvenes del Centro Cuatro Bocas, el porcentaje de jóvenes que califican en nivel alto o muy alto se incrementó de manera significativa. (Ver Figura 5).

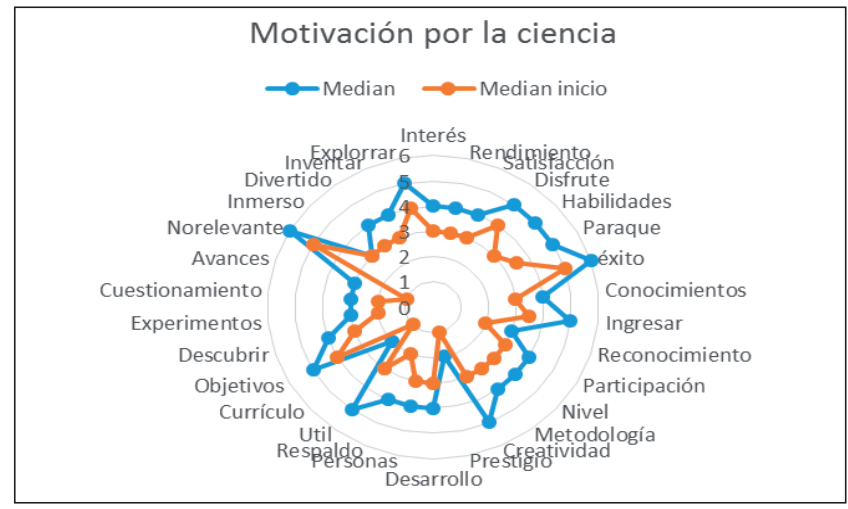

Figura 5. Motivación por ciencia

Fuente: autores 
El gráfico muestra diferencias en grado de motivación por el aprendizaje de la ciencia en las diferentes variables asociadas a la misma.

\section{Discusión}

Tal como lo plantea Gordon, (2001) la idea de estrategia induce a un abanico de posibilidades donde la creatividad del docente consiste en hacer un buen diagnóstico de las habilidades del grupo a quienes va a orientar, de los contenidos, del contexto y de selección de aquellas estrategias que en ese momento y para ese grupo son las más indicadas. La estrategia se determina como tal en la planificación, la puesta en escena y en la dinamización constitutiva de los procesos pedagógicos y didácticos antes, durante $\mathrm{y}$ después de la clase. (Atencia \& Garcia, 2013)

Los hallazgos concuerdan con la idea de que las instrucciones en el aula, inmersos en el factor producción, adquieren funciones que no pueden interpretarse limitativamente como discriminativas Goleman (2006); Gilar, Miñano, \& Castejón (2008); Cameron, (2002), sino que estas pueden clasificarse funcionalmente en términos de sus efectos en la adquisición de ejecuciones efectivas y su transferencia a situaciones aplicables, novedosas como desempeño inteligente y creativo.

Similar a Pabón, Nieto \& Gómez (2015), creatividad, es influida por prácticas pedagógicas de los profesores, recursos institucionales. Aunque existen factores socioculturales que influyen en el aprendizaje creativo contrario a López \& Tamayo (2012) si bien para el desarrollo de la creatividad de un sujeto cualquiera es de vital importancia conocer su interacción con el medio, factores de orden físico y socio-cultural no se asocian a la creatividad en jóvenes del centro educativo Cuatro Bocas. Acorde a Zambrano (2006) las nuevas ideas surgen cuando personas de diferentes disciplinas, experiencias y conocimientos colaboran en el proceso, coincidiendo en fases exploración y aplicación para estudiantes del Centro educativo Cuatro Bocas.

\section{Conclusiones}

Existen diferencias significativas en motivación antes y después de implementada metodología teniendo en cuenta factores como objetivo a trabajar en clase, interés por la ciencia, valor dado por la maestra.

Existen diferencias significativas en nivel de creatividad de los jóvenes al mejorar la motivación por el aprendizaje de las ciencias, considerándose el $99 \%$ de los estudiantes como jóvenes creativos (Jiménez, 1998). Creatividad se correlaciona con motivación e interés por asistir a clases de ciencias naturales. Habilidades en ciencias, aplicación de las ciencias a través de la experimentación (Sevillano, 2007), conocimiento a través de la invención, creatividad y diversión son factores asociados a motivación.

\section{Referencias}

Acevedo, C., Vergel, M. \& Flórez, E. (2012). Teoría de contacto aplicada al mecanismo Leva-palpador Cilíndrico, Bogotá. Editorial Universidad Nacional de Colombia.vol.1 pp.58.

Atencia Andrade, A., \& García Atencia, Corporación Universitaria del Caribe CECAR, Colombia, F. (2013). Incorporación de las tic en las metodologías de los docentes de especialización en docencia de cecar. Revista Logos Ciencia \& Tecnología, 5(1), 22-38. doi:http://dx.doi.org/10.22335/rlct. v5i1.3 SSN 1794-8231 SSN $2462-8794$ : $101-111$ 
Cameron, A. \& Keltner, D. (2002). The role of empathy in the formation and maintenance of social bonds. Behavioral and Brain Sciences, 25(01), 21 - 22.

Cascón, I. (2000). Análisis de las calificaciones escolares como criterio de rendimiento académico. Recuperado de: http://www3.usal. es./inico/investigacion/jornadas/jornada2/ comunc/cl7.html

Campos, A. (2009). Métodos mixtos de investigación. Lima: Investigar Magisterio

Dinello, R. (2006). Ludocreatividad y Educación. Bogotá: El Magisterio.

Gardner, H. (2001) Estructuras de la mente. Bogotá, Colombia: FONDO DE CULTURA ECONÓMICA LTDA.

Goleman, D. (2006). Inteligencia Social. Barcelona, España: KAIROS

Gordon, T. (2001). Maestro eficaz y técnicamente preparados. México: Diana.

Gilar, R. Miñano, P. \& Castejón, L. (2008). Inteligencia emocional y empatia: su influencia en la competencia social en Educación Secundaria Obligatoria. Tesis Doctoral. Universidad de Alicante, Alicante, España.

Huertas Díaz, O., Esmeral Ariza, S., \& Sánchez Fontalvo, I. (2014). La Educación en 110 Comunidades Indígenas; Frente a su proyecto de vida en un mundo globalizado. Revista Logos Ciencia \& Tecnología, 5(2), 232-243. doi:http://dx.doi.org/10.22335/rlct.v5i2.112

Jiménez, C. (1998) La Pedagogía de la creatividad y de la lúdica: Emociones, inteligencia y habilidades secretas. Bogotá: El Magisterio

Martin, J. (2011) Responsabilidad social universitaria: estudio acerca de los comportamientos, los valores y de la empatía en estudiantes de universidades iberoamericanas. Universidad de Valencia, Valencia, España.

Martínez, J., Vergel, M. \& Zafra, L. (2015). Comportamiento Juvenil y competencias prosociales, Bogotá, Ibáñez.

Ministerio de Educación Nacional. (2012). Nuevas tecnologías y currículo de matemáticas.

Mitjans, A. \& Díaz, A. (2013). Psicóloga Cubana, autora de muchos tratados sobre la conducta y la creatividad, 9(2), 427-434.

López, A. \& Tamayo, Ó. (2012). “Las prácticas de laboratorio en la enseñanza de las ciencias naturales". Revista Latinoamericana de Estudios Educativos, 1(8), 145-166

Ortiz, F. (2012). Evaluación, impacto en el aula. Revista Ecomatemático, 2(1).4-7.

Pabón Gómez, J., Nieto Sánchez, Z., \& Gómez, C. (2015). Modelación matemática y Geogebra en el desarrollo de competencias en jóvenes investigadores. Revista Logos Ciencia \& Tecnología, 7(1), 65-70. doi:http:// dx.doi.org/10.22335/rlct.v7i1.257

Richards, J., Platt, J. \& Platt, H. (1997). Diccionario de lingüística aplicada y enseñanza de lenguas. Barcelona: Ariel.

Rincón, O., (2011). Evaluación de actitudes hacia la incorporación de la Calculadora Voyage 200 en las aplicaciones de las ecuaciones diferenciales de primer orden. Revista Ecomatemático, 2(1), 21-26.

Ruiz, Y. \& Massa, M. (2011). Cambio significativo en la enseñanza de las ciencias, a través del uso del ordenador en la resolución de situaciones experimentales de fisica en el nivel universitario básico. Tesis doctoral. Universidad de Burgos, Burgos-España. 
Sánchez, J. (2010). Estudio comparativo de empatía médica, sensibilidad emocional $y$ características psicodemográficas entre estudiantes de Medicina y médicos especialistas profesores en Venezuela. Trabajo de grado. Universidad Autónoma de Madrid, Madrid- España.

Sanmartín, M., Carbonell, A. \& Baños, C. (2011). Relaciones entre empatía, conducta prosocial, agresividad, autoeficacia $y$ responsabilidad personal y social de los escolares. Tesis Doctoral. Universidad de Valencia, Valencia, España.

Sevillano, V. (2007). Empatía y cognición social en la preocupación por el medio ambiente. Tesis Doctoral. Universidad Complutense de Madrid, Madrid, España.

Vergel,M. (2015). Autoconceptoyjuventud: el papel de los contenedores de IBERCIENCIA. Iberoaméricadivulga: Organización de Estados Iberoamericanos. Recuperado de: http://www.oei.es/historico/divulgacioncienti fica/?Autoconcepto-y-juventud-el-papel

Vergel, M., Martínez, J., \& Duarte, H. (2015). Desarrollo del pensamiento matemático en estudiantes de cálculo integral su relación con la planificación docente. Revista Científica., $3(23), 17-29$

Vergel, M., Gallardo, H. \& Martínez, J. (2014). Factores asociados al rendimiento académico en estadística de estudiantes de administración pública. Bogotá: Colección Pedagogía Iberoamericana.

Vergel, M. \& Gallardo, H. (2007). Modelación en un museo interactivo. Imaginarios. X Reunión de la RED POP y IV Taller "Ciencia, Comunicación y Sociedad". Red de popularización de la Ciencia y la Tecnología en América latina y el caribe, San José, Costa Rica.
Vergel-Ortega, M., \& Largo-Leal, M.F. (2015). Em-pate em-patía: Emociones en el proceso de aprendizaje. Iberoaméricadivulga: Organización de Estados Iberoamericanos. Recuperado de: http://www.oei.es/historico/ divulgacioncientifica/?Em-pate-em-patiaEmociones-en-el

Vergel, M., Orjuela, J. \& Martínez, J. (2014). Modelos estimados para el tiempo de permanencia de estudiantes en asignaturas de Cálculo en la Universidad Francisco de Paula Santander. En XXVIII Reunión Latinoamericana de Matemática Educativa (Relme). Universidad del Atlántico, Barranquilla, Colombia.

Vergel Ortega, M., Martínez Lozano, J., \& Zafra Tristancho, S. (2015). APPS en el rendimiento académico y autoconcepto de estudiantes de ingeniería. Revista Logos Ciencia \& Tecnología, 6(2), 198-208.

Zafra, S., Martínez, J., \& Vergel, M. (2014). Indicadores para evaluar la pertinencia social en la oferta académica de programas. Revista Logos Ciencia \& Tecnología, 6(1), 165-177. doi: http://dx.doi.org/10.22335/rlct.v6i1.361

Zambrano, A. (2006). Los hilos de la palabra: Pedagogía y Didáctica. Bogotá: El Magisterio.
Enero-Diciembre 2016 ISSN 1794-8231 E-ISSN $2462-8794$ PP: 101-111 